\title{
SNR and BER Analysis for Multiple Antenna System Using OFDM
}

\author{
Aruba $\mathrm{R}^{1}$, Sinha G R ${ }^{2}$ And Rizwan S M ${ }^{3}$ \\ ${ }^{1}$ NationalInstitute of Technology, Delhi, India \\ ${ }^{2}$ Professor, ShrishankaracharyaTechnical Campus, BHILAI (C.G.)-490020, India \\ ${ }^{3}$ Professor, Caledonian (University) College Of Engineering, Sultanate of Oman
}

\begin{abstract}
Initially communication was carried out using analogue signal. Due to its various drawbacks, digital communication was introduced which has so many advantages over analogue communication and soon become very popular. Due to its low cost and easily reachable properties, wireless communication has become popular in communication.In the current scenario of modern wireless communication system, multiple input multiple output (MIMO) technique has emerged as one of the most efficient and significant method. This paper presents an analysis of MIMO system that has been introduced in the wireless communication to increase the diversity and to mitigate the effect of the channel fading phenomenon. In order to provide the transmit diversity, Space Time Block Code (STBC) is used in the Multiple input multiple output system. Effort has been made for deriving the Signal to noise ratio (SNR) and Bit error rate (BER) for different combination of the MIMO wireless communication system. SNR and BER have been computed for different combination of transmit antenna. The results of the simulation suggest that the performance of the MIMO system can be improved by increasing the diversity gain.
\end{abstract}

\section{Introduction}

In last few years, orthogonal frequency division multiplexing has proved to be most promising multiplexing technique in wireless communication system. The main reason behind the popularity of the OFDM is its bandwidth efficiency. Orthogonal frequency division multiplexing (OFDM) is more resistant to the ISI(Inter symbol interference) than any other multiplexing techniques. OFDM has been proved very efficient for both wired and wireless communication system.Systems which are used for navigational purpose to find out the location of the object on the earth or in space can also be considered as the wireless communication system. In almost all the wireless communication system, multicarrier modulation scheme is used for modulation of the signal and for transmitting the signal. Multicarrier modulation is the technique of the communication in which the transmitted data or signal is split in to a several component. Each of these separated components is then sent in separate carriers. The bandwidth of the individual carrier is narrow but the bandwidth of the composite signal is broader [1].In the current scenario, wireless communication technology is facing various challenges.Radio spectrum limitations, Complex nature of the wireless propagation environment and increasing demand of better quality service $(\mathrm{QoS})$ along with the higher data rate are some of the recent challenges. The effort has been made to make the wireless communication system spectrally more efficient by applying the clever coding techniques and algorithms. Due to the use of arrays of transmitting and receiving antenna at both the ends of the communication link, it offer much better spectral efficiency as compared to the traditional wireless system. In MIMO system all the arrays of transmitting and receiving antenna operates at the same frequency at the same time [2].

\subsection{MIMO System}

The arrangement of multiple antennas at transmitter and receiver side is called multiple-input and multiple-output (MIMO). There are three different categories of the MIMO system, first category improve the power efficiency by making the spatial diversity maximum. Delay diversity, STBC (Step time block code) STTC (Space time trellis code) are some of the variants of such technique. On the other hand, second category applies layered approach to increase the capacity. V-Blast is one of the popular schemes of such system in which full spatial diversity is not obtained. Finally the third category exploits the channel knowledge at the transmitter side [3].

\subsection{Orthogonal frequency division multiplexing (OFDM)}

Modulation is a phenomenon in which the information is mapped in to a change in frequency, phase or amplitude of the carrier signal. OFDMis a process of modulation and multiplexing combination. In this scheme, available bandwidth or resource can be shared among different individual modulated data sources. Amplitude Modulation, Frequency Modulation, phase modulation, BPSK(Binary Phase shift keying) and QPSK(Quadrature 
Phase shift keying) are some of the earlier and traditional modulation scheme in which only single carrier is used for modulation and information is modulated over a single carrier.OFDM, on the other hand is a multi-carrier modulation schemein which several carriers within the available bandwidth to send the information from source to the destination. In OFDM each carrier may use one of several available digital modulation schemes (BPSK, QPSK, QAM etc.)[5]. Delay spread is one of the undesirable characteristics of the wireless communication which causes the inter-symbol interference (ISI). Orthogonal frequency division multiplexing has proved to be very successful in mitigating the inter-symbol interference. It is due to this effect it has been adopted and used in many wireless communication system and various other standards [6-8].

\section{Literature Review}

It is very important to go through in the past work to gain some insight of its disadvantages, problems and remedy suggested by some researcher to overcome these problems of wireless communication. This section presents the past work done in the field of wireless communication.Seshadri et al. (1993) suggested two signaling schemes for improving the error performance of frequency-division-duplex (FDD) transmission systems using transmitter antenna diversity to improve the error performance of FDD (Frequency division duplex). Alamouti et.al (1998),proposed a simple transmit diversity technique for Wireless Communicationsused MRRC (Maximal ration receiver combining) with two transmitter antenna and one receiver antenna. This paper also explained that this scheme can also be generalized for making 2-transmitter and M-receiver wireless communication system. This scheme does not require any feedback information from the receiver to the transmitter. Bandwidth requirement is also not required in this scheme. Computational complexity of this system is also same as that of the MRRC. Zhou et. al. (2003)proposed a wireless communication system which was based on the STBC code (space time block code) transmission on the frequency selective fading.

Zeng et al (2006) studied the performance of combination of two coding scheme. In this appear an extensive study has been carried out by concatenating the outer channel code with OSTBC (Orthogonal space time block code). In this study, convolution code or trellis -coded modulation code is used for outer coding. They also derived the upper bound on bit error rate for this combination coding scheme. They showed that diversity order with antenna selection remain almost same as that of full complex system. But deterioration of SNR is upper bounded by $10 \log _{10}(\mathrm{M} / \mathrm{L}) \mathrm{dB}$.Salah, et.al (2007)investigated the linear pre-coding of non-orthogonal STBC (Space time Block code). This first of all derived the theoretical model of spatial correlation with Laplacian distribution for AOA. A code word error matrix which fulfilled the required criterion is used to design the pre-coder. Author's proposed a criterion for selecting the suitable code word error matrix. This criterion was based on the probability of the system outage. The selected codeword error matrix moves very rapidly from one diversity order to another. The selection of suitable codeword suggests the vitality of the determinant and Eigen values of error matrix. They tested this method to non orthogonal optimal STBC structure of $2 \times 2,4 \times 4$.Jia, et al. (2012) presented a generalised spatial modulation (SM) scheme with multiple active transmits antennas, named as multiple active-spatial modulation (MA-SM). By allowing multiple transmitting antennas in the SM system for transmitting different symbols at the same time instant, MA-SM takes advantages of the low complexity of SM and high multiplexing gain of Vertical-Bell Lab Layered Space-Time (V-BLAST) system. In the MA-SM system, the transmitted symbols are mapped into a high dimensional constellation space including the spatial dimension. A closed form bound for the bit error probability (BEP) of the proposed detection scheme is also derived in this work. Numerical results with the comparison among the existing multiple-input multiple-output (MIMO) systems such as space time block code (STBC) and V-BLAST exhibit the effectiveness and performance of MA-SM.Fang, et al.(2015)analyzed the performance of the protograph low-density parity-check (LDPC) codes concatenated with space-time block code (STBC) on Rayleigh fading channels. In this work, authors first extend the modified protograph extrinsic information transfer (PEXIT) algorithm for analyzing the convergence performance of protograph codes. On the basisof the extended PEXIT algorithm and Gaussian approximation, authors further derive the asymptotic bit-error-rate (BER) expression of protograph codes. Utilizing the PEXIT algorithm, theoretical and simulated BERs is obtained,then compared the performance of two classical protograph codes, i.e., accumulate-repeat-by-3-accumulate (AR3A) code and accumulate-repeat-by4-jagged-accumulate (AR4JA) code, regular LDPC code, optimized irregular LDPC codes, and demonstrate that the AR3A code is superior to other three codes. moreover, authors discussed the impact of the number of receive antennas (i.e., NR) on the system performance and verified that the theoretical analyses hold as NR varies. thus, the AR3A code stands out as a good candidate for wireless communication applications with multiple antennas.Hosseiniet.al (2015)suggested a comprehensive study and analysis on the Full-Rate and Linear-Receiver (FRLR) STBC proposed as a newly coding scheme with the lower decoding complexity for a $2 \times 2$ MIMO system. It is shown that the FRLR code suffers from the lack of the non-vanishing determinant (NVD) property that is a important parameter in designing a full-rate STBC with a good performance in increasing data rate, across QAM constellation. To overcome this disadvantage, the existence of the NVD feature for the FRLR code depends on the type of the modulation used. In particular, it has been analytically proved that the FRLR code fulfils the NVD 
property across the PAM constellation but not for the QAM scheme. Simulation results reveals that, at a BER equal to $10^{-4}$, utilising the PAM modulation for the FRLR-STBC, gives about $2 \mathrm{~dB}$ gain over a use of the QAM when the bandwidth efficiency is $6 \mathrm{~b} / \mathrm{s} / \mathrm{Hz}$. In addition, for the PAM constellation, the FRLR code significantly outperforms several existing full-rate STBCs. Finally, we have utilized the moment generating function a method to derive an exact closed-form expression for the average error probability for the FRLR code with the BPSK modulation.

Carrier frequency offset (CFO) attenuate the signal amplitude and produce interference between the carriers. The imperfection or mismatch in the local oscillator causes the phase noise which also has adverse effect in the signal. An unsynchronized clock causes the timing error which hence causes imperfect sampling of the signal which leads to the signal error. Propagation delay produce due to the IC's also responsible for the timing error.Single antenna system requires very large bandwidth for data transmission so data transmission rate is much less in single antenna communication system than the multiple input multiple output (MIMO) system. In MIMO, OFDM is used for modulation of the signal which requires less bandwidth but OFDM also exhibit the Inter symbol interference (ISI) which makes the receiver difficult to extract the actual signal from the received signal. STBC is able to achieve the transmitter diversity along with providing the antenna gain but fail to produce code gain. Space time trellis code is able to achieve full diversity along with coding gain but it is basically complex in decoding and cost.Further to this, while studying analysis of multiple antenna system using OFDM in term of SNR and BER by Gulshan and Sinha (2016), some interesting results of the simulation showing the improved performance of the MIMO system are also obtained in this paper. Results are shown on SNR Vs CFO, SNR Vs Phase Noise, SNR Vs BER for OFDM system and SNR Vs BER for 2:1 MIMO system.

\section{Proposed Method}

Severe attenuation in a signal during the transmission is one of the crucial problems in the wireless communication which need to be handled carefully. One of the remedy of this problem is space time block code which is designed and develop by keeping the multiple antenna system into consideration. The main aim of this work is to perform the analysis of the Signal to noise ratio (SNR) by changing the carrier frequency offset(CFO), time jitter and phase noise. Analysis has been carried out with the help of graph developed in the MATLAB environment. One more graph has been used which BER versus for analysis purpose. In the next section the mathematical expression for the noise, time jitter, and CFO has been derived. A mathematical expression for SNR (Signal to Noise ratio) has also been derived in term of phase noise, time jitter and CFO, number of subcarriers $(\mathrm{N}), \mathrm{SNR}$ of input $\left(\mathrm{Y}_{\mathrm{in}}\right)$ and attenuation/gain $(\alpha)$.

\subsection{Calculation of SNR and BER}

The transmitted OFDM signal for the $\mathrm{m}_{\mathrm{th}}$ symbol is given by the $\mathrm{N}$ point complex modulation sequence:

$$
x_{m}(n)=\sum_{k=0}^{N-1} X_{m}(K) e^{\frac{j 2 \pi n k}{N}}
$$

where $\mathrm{n}$ ranges from 0 to $\mathrm{N}+\mathrm{Ng}-1$. Bit Error Rate can be determined with the help of $\mathrm{E}_{0} / \mathrm{N}_{0}$ :

$$
B E R=0.5 * \operatorname{erfc}(\sqrt{(S I N R)})
$$

\subsection{STBC-OFDM}

The block diagram of the proposed methodology is shown in Fig. 1, Gulshan and Sinha (2016) which represents the MIMO system with four transmitters and one receiver. For the two transmitters and single receiver and for the six transmitters and the single receiver is same with only change in the transmitter section where number of transmitter antenna will be 2 and 6 for 2:1 MIMO system and 6:1 MIMO system.

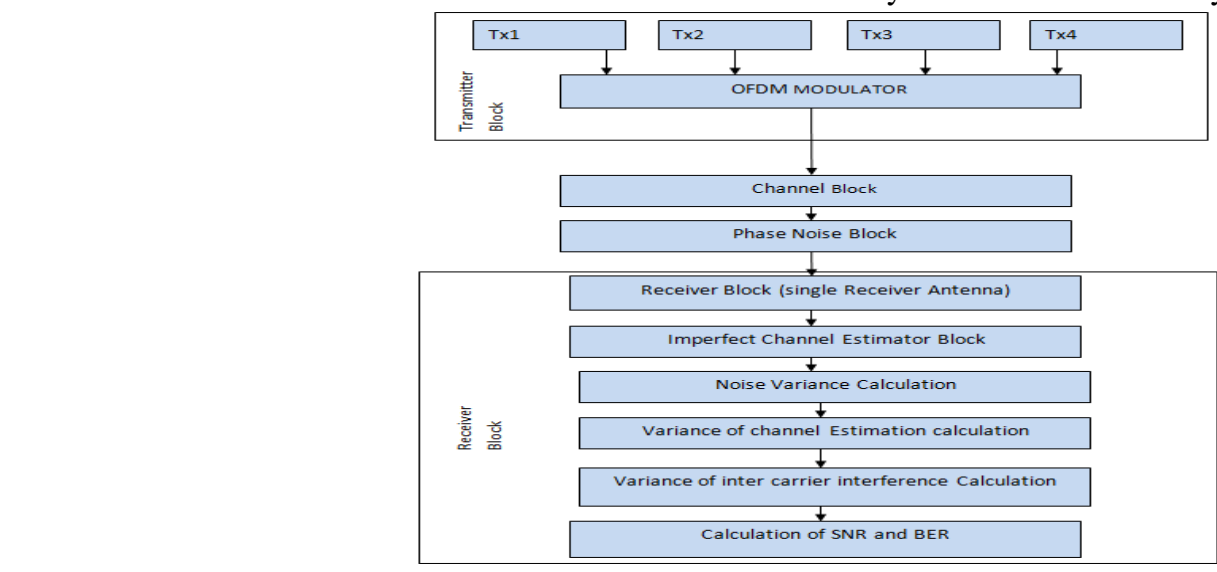




\section{Figure 1: Proposed MIMO}

Transmitter module or block consists of four numbers of antenna and the OFDM modulator. In the first time instant symbol[Xo, X1, X2, X3] are transmitted simultaneously from four transmitting antenna with X0 is transmitted through antenna1 X1 is transmitted through antenna 2 and so on. In the second time slot, symbols [$\left.\mathrm{X} 1 *, \mathrm{X} 0^{*}, \mathrm{X} 3^{*}, \mathrm{X} 2 *\right]$ are transmitted. Similarly symbols $\left[-\mathrm{X} 2 *,-\mathrm{X} 3^{*}, \mathrm{X} 0^{*}, \mathrm{X} 1 *\right]$ and $[\mathrm{X} 3,-\mathrm{X} 2,-\mathrm{X} 1, \mathrm{X} 0]$ are transmitted during the third and fourth time slots respectively.For each transmit antenna, a block of $N$ complexvalued data symbols $\{X(k)\}$ for $\boldsymbol{k}=\mathbf{0}$ to $N-1$ are grouped and converted into a parallel set to form the input to the OFDM modulator, where $k$ is the sub carrier index and $N$ is the number of sub carriers. The modulator consists of an Inverse Fast Fourier transform (IFFT) block. The output of the IFFT at each transmitter is the complex baseband modulated OFDM symbol in discrete time domain and is computed by using below mention formula

$\mathrm{x}(k)=\frac{1}{\sqrt{N}} \sum_{k=0}^{N-1} X(k) e^{\frac{j 2 \pi k n}{N}} ; 0 \leq n \leq N-1$

Where $\mathrm{k}=$ sub carrier index, and $\mathrm{N}=$ number of sub carrier. Channel for this project is modeled by a tapped delay line. Channel coefficients in the tapped delay lines are so selected that it is assumed to be slowly varying and hence can be considered as constant over the transmission instant. In the channel module, channel frequency response for $k^{\text {th }}$ subcarrier is given by

$$
\mathrm{H}(k)=\sum_{p=0}^{L-1} h(p) e^{\frac{j 2 \pi p k}{N}}
$$

Receiver module takes the received signal and performs the channel estimation and variance of noise, variance of channel estimation and variance of inter-carrier interference.Once all the bits of the transmitted signal have been estimated then signal to noise ration and Bit error rate has been computed to check the performance of the proposed methodology. This module is designed to perform this function. In this project, 16 QAM modulationsare used with gray code mapping for the signal (b0b1b2b3). Bit error rate is given by

$p_{e}=\frac{1}{2} * \int_{0}^{X}\left[p_{e}(b 1 \mid H 0, H 1, H 2, H 3, H 4, H 5)+p_{e}(b 3 \mid H 0, H 1, H 2, H 3, H 4, H 5)\right] P(\gamma) d(\gamma)$

\section{Experimental Results}

In order to simulate the Proposed method, first of all a simulation program has been designed for the proposed MIMO (Multiple I/O) system. This system of MIMO is designed by taking number of antenna as 1, 2, 4 and 6 while keeping the number of receiver one. Proposed testing system is designed in MATLAB platform, the system consists of Transmitter module, receiver Module, Channel module etc. For modelling the transmitter module, first of all OFDM modulator is designed which takes the transmitted signal from transmitter (it may be $1,2,4,6)$, modulate the signal and send it to the channel. It is to be noted here that OFDM modulator is designed separately for the entire transmitter. In order to put the effect of the channel on the transmitted signal from all the transmitter, channel module has been designed which add some random as well as the phase noise in the signal. This channel module is defined differently for different transmitted signal which add the effect of channel differently in different transmitted signal.Receiver block consist of various sub-blocks which has been described in the last section. Channel estimator sub block of the channel module estimate the channel on the basis of receive signal and get the estimated copy the transmitted signal along with other processing operation. The whole simulation program is accompanied with the STBC (Space Time Block Code) code for performance improvement of MIMO system. Table 1 shows the systems parameters used.

Table 1: System Parameters.

\begin{tabular}{|c|l|}
\hline Parameters & Values \\
\hline$\sigma h^{2}$ & $0.1,0.2,0.02,0.4$ \\
\hline$\sigma \varepsilon^{2}$ & $0.1,0.04,0.06$ \\
\hline Subcarriers(N) & 64 \\
\hline Channel path gains & $-9.7,-0.9,-8.5,-0.5$ \\
\hline
\end{tabular}

A simulation program was designed for different combination of antenna system by incorporating the STBC code. Output of the simulation program is shown in the Fig. 2 and Fig. 3. The analysis has been performed on the effect of the signal to noise ratio by varying the carrier frequency offset (CFO), phase noise and time jitter. 


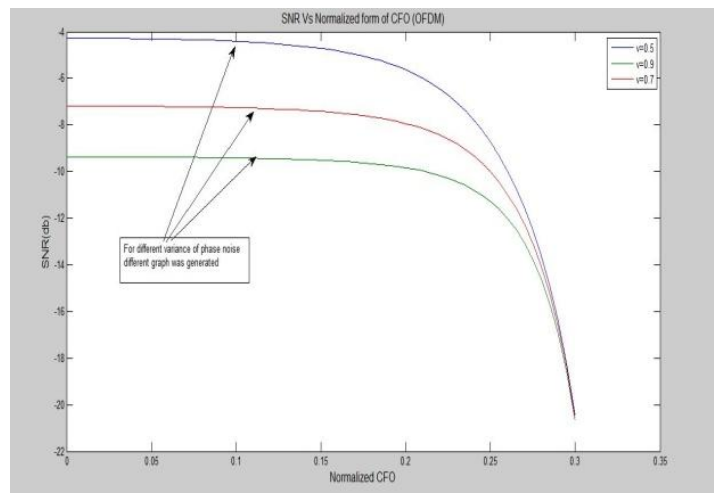

Figure 2: SNR Vs CFO for non STBC-OFDM system.

Figure 2 depicts that SNR (dB) and X-axis denotes the normalized Carrier Frequency Offset (CFO). It is observed that SNR is high when Carrier Frequency Offset is zero. SNR is exponentially decreasing. There are three curves in the figure for different variance of phase noise. For plotting the above graph we fixed the value of variance of phase noise and after that by varying or repeating the value of carrier frequency offset value.

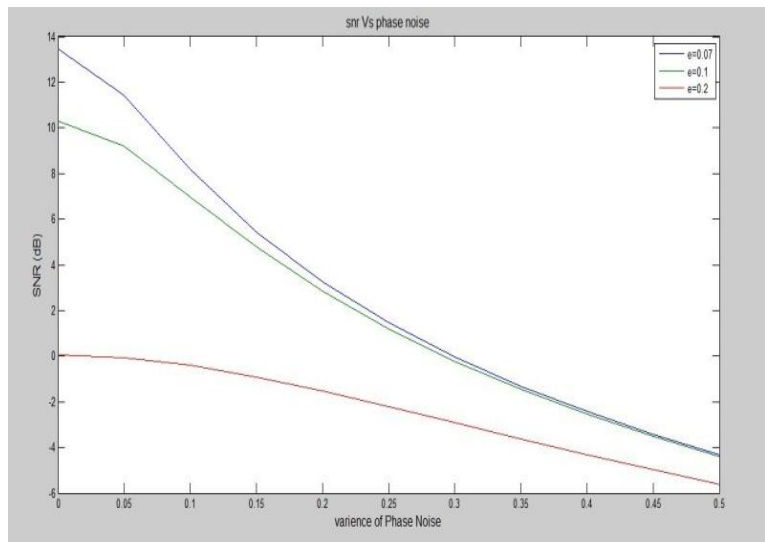

Figure 3: SNR Vs phase noise for non-STBC OFDM system

The SNR decreases with increasing value of the phase noise. There are three curves for three different value of CFO (e). The curve is decreasing if the value of CFO is increasing. For plotting the above graph we fixed the value of carrier frequency offset and after that by varying or repeating the value of variance of phase noise value. Fig. 4 shows SNR versus BER plot.

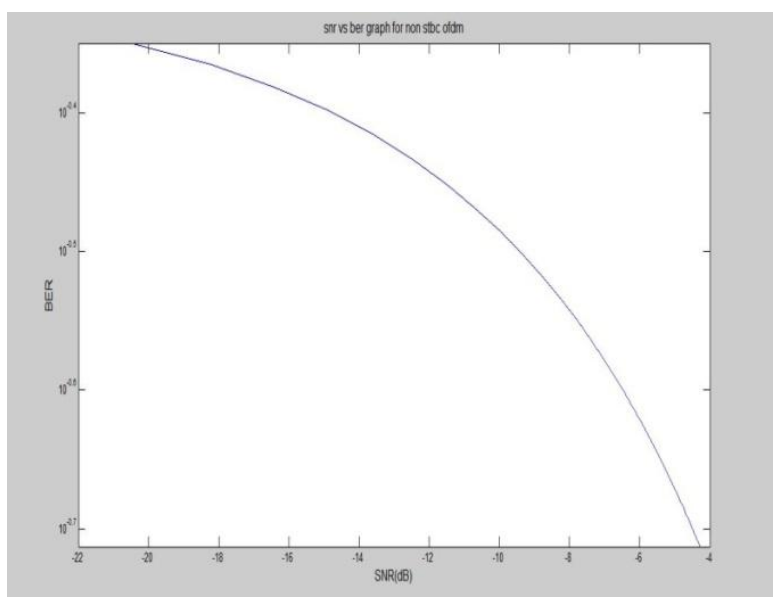

Figure 4: SNR Vs BER graph for OFDM system.

For this simulation we use three different values of phase noise and for this highest SNR value the BER is decreasing fast compared to the other value of $\mathrm{v}$ and corresponding SNR.Fig. 5 shows the SNR Vs BER curve for different values of phase noise 2x1 transmission systems. X-axis represents the SNR (db) and y-axis denotes 
the bit error rate.From the graph it has been seen that for the value of phase noise $\mathrm{Ve} 0=0.3$, we get highest SNR curve. For this simulation we use three different values of phase noise and for this highest SNR value the BER is decreasing fast compared to the other value of $v$ and corresponding SNR.

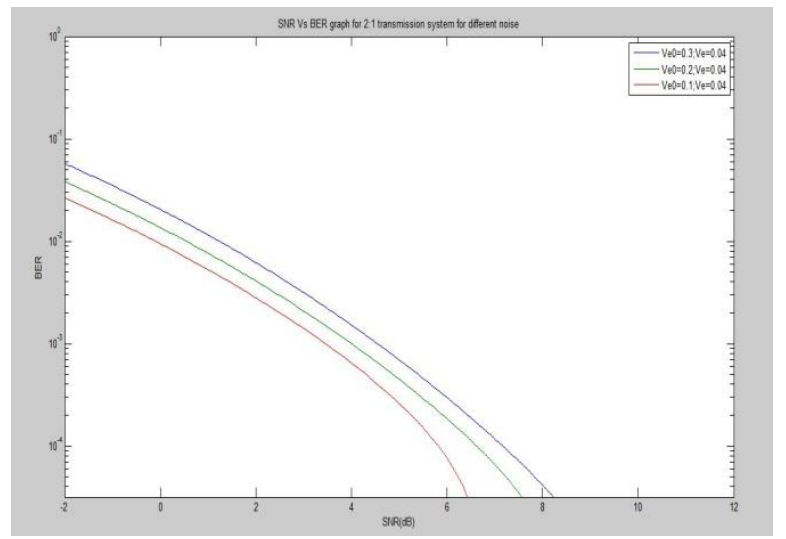

Figure 5: SNR Vs BER graph for different phase noise for two transmitter MIMO system.

\section{Conclusions}

Main reason for loss in performance of wireless communication is fading. In order to overcome this problem of wireless communication, engineers came up with a new technique which is called diversity. This technique basically reduces the probability of getting an overall weak channel. The term diversity describes the different dimension of the channel for example, space, time, frequency etc., to improve the channel experienced by the receiver. In this paper, a realization of the space time block code has been carried out considering various effect of the wireless communication.A space time code can be considered as the modulation scheme which is designed and developed for the MIMO wireless communication system. An attempt has been made to derive the SNR (signal to noise ratio) and BER(Bit error rate) for different combination of MIMO system. What impact, a noise, carrier interference and channel response produces on the MIMO system has been designed, developed, simulated and then analyzed. SNR Vs BER curve indicate that the performance of the MIMO system can be improved by increasing the diversity gain.The SNR Vs BER curve shows that the performance of the system is improvedon increasing the diversity gain.MIMO architecture has been studied and analyzed.

\section{References}

[1]. J. G. Proakis, Digital Communications, McGraw Hill, New York, 2001, pp. 822-823.

[2]. J. J. van de Beek, O. Edfords, M. Sandell, S. K. Wilson, and P. O. Börjesson, "Channel Estimation in OFDM Systems," in Proc. 45 IEEE Vehicular Technology Conf., Chicago, IL, July 1995, pp. 815-819.

[3]. S. Coleri, M. Ergen, A. Puri and A. Bahai, "Channel Estimation Techniques Based on Pilot Arrangement in OFDM Systems," IEEE Transactions on Broadcasting, Vol. 48, September 2002, pp. 223-229.

[4]. Michel C. Jeruchim, Philip Balaban and K. Sam Shanmugan, Simulation of Communication Systems, , Kluwer Academic / Plenum Publishers, New York, 2000, pp. 545-621.

[5]. N. Seshadri and J. H. Winters, "Two signaling schemes for improving the error performance of frequency-division-duplex (FDD) transmission systems using transmitter antenna diversity," in Proc. IEEE Veh Technol. Conf. (VTC), Secaucus, New Jersey, USA, May 1993, pp. 508-511.

[6]. Alamouti, Siavash M. "A simple transmit diversity technique for wireless communications." IEEE Journal on Selected Areas in Communications, 16.8, 1998, pp. 1451-1458.

[7]. Zhou, Shengli, and Georgios B. Giannakis. "Single-carrier space-time block-coded transmissions over frequency-selective fading channels." IEEE Transactions onInformation Theory, 49.1, 2003, pp. 164-179.

[8]. X. N. Zeng and A. Ghrayeb, "Performance bounds for combined channel coding and space-time block coding with receive antenna selection" IEEE Trans. on Veh. Technol., vol. 55, no. 4, July 2006, pp. 1441-1446.

[9]. Abdellatif Salah, "On the linear precoding of non-orthogonal STBC for correlated MIMO channel" The 18th Annual IEEE International Symposium on Personal, Indoor and Mobile Radio Communications (PIMRC'07), 2007.

[10]. HemlataSinha and G.R.Sinha, "Performance of Linear Block Coding for Multipath Fading Channel" I.J. Information Technology and Computer Science, July 2012.

[11]. HemlataSinha and G.R.Sinha, "Comparative Study of Rayleigh Fading Multiple Antenna System with MRC" Mathematical Methods and Systems in Science and Engineering ISBN: 978-1-61804- 281-1, April 2015.

[12]. Jintao Wang, ShuyunJia, and Jian Song, "Generalised Spatial Modulation System with Multiple Active Transmit Antennas and Low Complexity Detection Scheme" IEEE Transactions on Wireless Communications, Vol. 11, No. 4, April 2012.

[13]. Fang, Yi, "Asymptotic performance analysis of protograph LDPC-coded STBC systems in fading channels" IEEE 26th Annual International Symposium on Personal, Indoor, and Mobile Radio Communications (PIMRC), 2015.

[14]. Hosseini, Seyyed Saleh, SaharTalebi, and JamshidAbouei, "Comprehensive study on a $2 \times 2$ full-rate and linear decoding complexity space-time block code." Communications, IET 9.1, 2015, pp.122-132.

[15]. GulshanJaiswal and G.R.Sinha, "Analysis of Multiple Antenna System using OFDM in terms of SNR and BER" International Journal of Advance Research in Engineering, Science \&Technology, Vol.3, Issue 5, May 2016, pp. 736-746. 